\title{
THE CENTRIFUGE METHOD OF DETERMINING PROTOPLASMIC VISCOSITY
}

\author{
L. V. HEILBRUNN \\ Zoölogy Department, University of Michigan
}

The study of protoplasm from a physical standpoint must always depend on the possibility of making measurements of its physical properties. Such measurements are in most instances difficult, often they are extremely inexact and uncertain.

Fortunately, it is now possible to determine the viscosity, or its inverse the fluidity, of at least certain types of cells. Methods of viscosity determination have been devised both for plant and for animal cells. In dealing with animal cells, the centrifuge method is certainly the surest and the most accurate. It is therefore thought advisable to publish further details in regard to this method in the hope that it may find wider application.

The main principles underlying the use of the centrifuge method have already been described(1). Measurements in the past have been made with isolated cells-egg cells or protozoa. These are introduced with a fine pipette into narrow glass tubes which are of a size to fit conveniently into the haematocrit attachment of an ordinary hand centrifuge. With a centrifuge of the Bausch \& Lomb type it is best to use tubes about $4 \frac{1}{2} \mathrm{~mm}$. in outside diameter and $3 \mathrm{~mm}$. or a trifle more than $3 \mathrm{~mm}$. inside diameter. It is important to have the glass clear and free from glass dust. The ends of the tubes are sealed in a flame some time before they are ready for use. This can be done very rapidly and it is indeed simpler to make new tubes than to clean the old ones. A large supply of tubes is needed and care must be taken not to mix 
the tubes of various sets. The tubes of any given pair should be of approximately equal weight. A convenient practice, which insures tubes of approximately equal weight and which prevents mixing of sets of tubes, is to cut off lengths of tubing a little longer than twice the length suitable for one end of the haematocrit attachment. Both ends of this double tube are then sealed in a flame. A pair of tubes are cut from its ends when they are needed. Since the two tubes are from an adjacent strip of glass tubing, they can be assumed to be of about the same weight, and as they remain together until needed there is no chance of confusing various sets of tubes.

For many cells a convenient rate of speed with the hand centrifuge is one turn of the high-speed handle per second. With the Bausch \& Lomb centrifuge this represents a speed of 130 revolutions per second. The speed with other models of hand centrifuge can be easily determined by turning the handle slowly and counting how many turns of the tubes accompany a complete turn of the handle. It is, of course, best to turn the handle evenly, and the turning should be controlled by a stop-watch, but in practice slight variations in rate of turn are of no significance. For very viscous protoplasm turning with a hand centrifuge may become wearisome. In such cases a water centrifuge or an electric centrifuge can be used, and the number of revolutions per second is then determined with the aid of an odometer or speed indicator such as may be purchased from various supply houses.

A measure of the viscosity is obtained by noting the number of seconds necessary for the granules to move through a given part of the cell. In ciliated protozoa particles of oil or other material can be introduced into the cell and their speed noted. Ordinarily a relative measure of the viscosity is all that is needed. The viscosity of the protoplasm under one set of conditions is compared with the viscosity of the protoplasm under another set of conditions. But it is also possible with the centrifuge method to obtain a measure of the absolute viscosity of the protoplasm. Such measurements have been made and will be published shortly. 
In determining the absolute viscosity, the following form of Stokes' law is used:

$$
V=\underset{9 \eta}{2 \operatorname{cog}(\sigma-\rho) a^{2}}
$$

In this formula $V$ is the speed of movement of the granules in the cell and is determined by observation; $c$ is the centrifugal force in terms of gravity and can be computed easily(1); $g$ is the gravity constant; $a$, the radius of the granule; $\eta$, the viscosity. The term o- $\rho$ requires further comment; $\rho$ is the specific gravity of the granules; $\sigma$, the specific gravity of the fluid through which they move. It is possible to determine o by breaking up the cells with splintered glass and then centrifuging the granules in different solutions of known specific gravity. When the specific gravity of the solution is less than that of the granules, there is an accumulation of granules at the bottom of the centrifuge tube; when the specific gravity of the solution is greater, there is an accumulation at the top. By making a series of tests the specific gravity of the granules can be shown to lie between two close limiting values. o cannot be determined directly. But an approximate value can be obtained on the basis of simple calculations if the specific gravity of the cell is known. It is easy to determine the specific gravity of the cell in the same way that the specific gravity of the granules is determined. When the values for $V, c, g, \odot, p, a$, are substituted into the formula, it can be solved for the viscosity, $\eta$.

The centrifuge method can be used not only on isolated cells, but also on cells in mass. It is, of course, possible to centrifuge entire animals, and the tissues of such animals can then be examined cytologically. The possibilities of such a procedure are now being investigated. It seems certain that, at least in some types of cells, it will be practicable to determine the approximate viscosity of the protoplasm of a cell while it is within the animal. It even appears probable that a rough determination can be made of the viscosity of the nuclear material. 
One great advantage of the centrifuge method lies in the fact that it does not involve the introduction of any foreign material into the cell. This is extremely important. All cells are certainly very sensitive to injury at their surfaces, and it is hard to conceive of any rupture of the outer wall without a more or less decided injury to the interior. In the centrifuge method the granules normally present in the cells are the only materials necessary for viscosity measurement. Their movement can have little effect on cell life, for it has repeatedly been found that centrifuged cells live and show no signs of injury.

Many authors have accepted the measurements of viscosity made with the centrifuge. Weber(2), in his survey of methods used in determining protoplasmic viscosity, mentions no other type of measurement for animal cells, he classes the microdissection method as a 'Schätzungsmethode,' that is to say, an 'estimation method.' Recently, Chambers, whose results with microdissection do not always accord with the findings obtained with the centrifuge, has pointed out what he regards as objectionable features of the centrifuge method(3). He says, first, that it is too drastic and, second, that too long a time intervenes between the centrifugal test and observation under the microscope.

It is hard to understand what Chambers means by his second criticism. The cells are removed from the centrifuge and are generally examined within a few seconds after the completion of the centrifugal turning. But whether a few seconds, or many seconds, or even many minutes intervene between the centrifugal test and the microscopical observation, the results are always the same. The granules moved by the centrifuge do not return quickly to their original position.

Nor can it be assumed that the centrifuge method is drastic. It has repeatedly been shown that egg cells which have been centrifuged continue to develop normally. The centrifuge method is certainly far less drastic than the microdissection method, for eggs subjected to microdissection presumably do not continue to develop normally. They are oftentimes very severely injured. 
Chambers apparently believes that the movement of the granules during centrifugal treatment produces a change in viscosity while the measurement is in progress. It would seem offhand that such a criticism could not bear very great weight, for the movement of the granules in centrifuged cells is usually quite slow, not so much faster in some instances than the normal wanderings of the granules in untreated cells. Add to this the fact that until the present the centrifuge method has been concerned only with relative measurements. For such measurements, even if it is granted that the centrifuge method affects the viscosity of the protoplasm, the effect would be about the same on one occasion as on another, so that the end result would be the same when the two values are compared.

But there is perhaps a certain amount of point to Chambers' criticism. It is a criticism that may be applied to every type of viscosity measurement both in living and lifeless fluids, and to some other types of physical measurement as well. No matter which method of viscosity determination is used, there is always a possibility that the method itself may induce an alteration of viscosity while the measurement is in progress. Although this point is often overlooked by the physical chemist, it is clearly recognized by Drucker(4).

It is to be expected that some methods of viscosity determination would have a greater effect than others on the viscosity of the fluid being measured. It is quite possible that the microdissection method would have a marked effect even in addition to the direct effect of an injury at the surface. In the case of the centrifuge method, it can be readily shown that there is no appreciable effect of the measuring device.

If centrifuge measurements are made at varying speeds, that is to say, at various degrees of centrifugal force, any effect that might be produced on the fluidity of the protoplasm by the movement of the granules would be greater the higher the speed of the moving granules. For with greater speed the momentum of the granules would be greater. Thus it might be supposed that the moving granules had the power of 
breaking up some structural elements in the protoplasm and of thus increasing the fluidity. The power of the granules to accomplish such a breakdown of structure with resultant liquefaction would depend not so much on the time during which the granules moved as upon their momentum. If, therefore, the granules exert a marked liquefying action, it is to be anticipated that such action would be very much more pronounced at higher speeds of the centrifuge, that is to say, under conditions in which the granules move faster. But by actual test it has been found that the viscosity determinations made at low centrifugal speed are very close to those made at higher speed, so that it can safely be assumed that the movement of the granules is of no consequence in altering the viscosity values as determined by the centrifuge method.

If we examine the formula on page 315 , it will be noted that as the centrifugal force, $c$, is increased, there is obviously also an increase in the velocity of granular movement, $V$. The other quantities, $g, o, \rho, a$, do not change. Thus if the viscosity, $n$, remains constant, we should find that as we increase the centrifugal force, $c$, there is an exactly proportional increase in the velocity of the granular movement, $V$. If there is no such proportionality, then obviously the viscosity, $n$, must change as we vary the centrifugal force. On the other hand, if $V$ is directly proportional to $c$, then it is clear that the movement of the granules has no effect on the viscosity.

As is well known, the centrifugal force varies as the square of the number of turns of the centrifuge per second. As we increase the speed of centrifugal turn, the speed of granular movement is found to increase as the square of the number of turns of the centrifuge per second. This is shown by the following table, which gives the results of a series of measurements on Cumingia eggs. The first column of this table, labeled $l / n$, gives the number of seconds required in a given test to complete a single turn of the high-speed handle of the centrifuge. It is therefore inversely proportional to the speed of turn of the centrifuge. The second column gives the 
time in seconds necessary for the dark granules of the egg to move far enough so that they form a definite zone at one end of the egg. This, therefore, is in inverse ratio to the speed of movement of the granules, i.e., it is $\mathrm{l} / \mathrm{V}$. In the third column, $\mathrm{l} / \mathrm{V}$ is calculated for the one-, three-, and four-second values of $\mathrm{l} / \mathrm{n}$, from the figure found for the two-second value. The fourth column gives the time required for both the dark granules and the pigment granules of the Cumingia egg to move to such an extent that three distinct zones can be recognized. These figures again represent an inverse of the speed of granular movement, and they are shown in the table as $1 / V^{\prime}$. In the fifth column the values of $1 / V^{\prime}$ are calculated for the one-, three-, and four-second values of $1 / \mathrm{n}$ from the figure found for the two-second value. It will be seen at a glance that the second and third columns and the fourth and fifth columns agree very closely. The agreement is not so close for the one-second value of $\mathrm{l} / \mathrm{n}$, but this particular determination is subject to a large experimental error. When the centrifuge is turned at the rapid rate of one turn per second, the number of turns required to produce the required granular movement is small. Under these conditions, it is difficult to turn the centrifuge exactly two times or three times, for the centrifuge cannot be brought to a stop immediately after the turning is completed. The agreement for the other values of $1 / n$ is strikingly close.

\begin{tabular}{|c|c|c|c|c|}
\hline $1 / n$ & $1 / V$ & 1/V CALCULATED & $1 / \mathrm{V}^{\prime}$ & 1/ $\mathrm{V}^{\prime}$ CALCULATFI \\
\hline 1 & 2 & 3 & 3 & $3 \frac{1}{2}$ \\
\hline 2 & 12 & 12 & 14 & 14 \\
\hline 3 & 27 & 27 & 33 & $31 \frac{1}{2}$ \\
\hline 4 & 48 & 48 & 56 & 56 \\
\hline
\end{tabular}

The table proves definitely that the speed of granular movement is proportional to the square of the number of turns per second of the centrifuge. That is to say, it is proportional to the centrifugal force. As will be understood from the previous argument, this could not be the case if the vis- 
cosity of the protoplasm varied as the magnitude of the centrifugal force varied. Thus the viscosity of the protoplasm is entirely independent of the magnitude of the centrifugal force. This is definite proof that the movement of the granules through the cytoplasm has no appreciable effect on the protoplasmic viscosity.

\section{SUMMARY}

The methods involved in making determinations of protoplasmic viscosity by the centrifuge method are described.

Evidence is presented to show that the movement of granules through protoplasm has no effect on the viscosity measurement.

\section{BIBLIOGRAPHY}

1 Heilbrun 1921 Jour. Exp. Zoöl., vol. 34, p. 417.

2 WeBer 1924 Methoden der Viscositätsbestimmung des lebenden Protoplasmas, in Abderhalden's Handbuch der biologisehen Arbeitsmethoden, Abt. XI, Teil 2. Berlin and Vienna.

3 Chambers 1924 The physical structure of protoplasm as determined by micro-dissection and injection. In General Cytology, edited by E. V. Cowdry, Chicago.

4 DRUCKER 1917 Zeitsch. f. physik. Chem., Bd. 92, S. 287. 